\title{
Fuzzy Membership, Possibility, Probability and Negation in Biometrics
}

\author{
Nicolaie Popescu-Bodorin ${ }^{\dagger}$, Valentina E. Balas \\ ${ }^{\dagger}$ University of S-E Europe Lumina, Colentina 64B, 021187, Bucharest, Romania. \\ * ‘Aurel Vlaicu’ University, Bd. Revolutiei 77, 310130, Arad, Romania. \\ †popescu.bodorin@lumina.org, *valentina.balas@uav.ro
}

\begin{abstract}
This paper proposes a new formalization of the classical probability-possibility relation, which is further confirmed as a much complex, but natural provability reachability - possibility - probability - fuzzy membership - integrability interconnection. Searching for the right context in which this relation can be consistently expressed for the particular case of experimentally obtained iris recognition results brought us to a natural (canonic) and universal fuzzification procedure available for an entire class of continuous distributions, to a confluence point of statistics, classical logic, modal logic, fuzzy logic, system theory, measure theory and topology. The applications - initially intended for iris recognition scenarios - can be easily extrapolated anywhere else where there is a need of expressing the relation possibility - probability - fuzzy membership without weakening the $\sigma$-additivity condition within the definition of probability, condition that is considered here as the actual principle of possibility-probability consistency.
\end{abstract}

Keywords: $\sigma$-additivity, principle of possibility-probability consistency, provability, reachability, possibility, probability, fuzzy membership, Riemann integrability, negation, consistent experimental frameworks, Turing test, iris recognition, biometrics.

\section{Introduction}

Implementing biometric identification systems means advancing from human intuition to artificial but formally correctly biometric decisions. To be specific, when a human agent analyzes a pair of two good quality iris biometric samples (two iris images) - for example, it is easy for him to decide if the pair is a genuine or an imposter one, hence it is simple for him to have the intuition that designing a reliable artificial agent able to recognize genuine against imposter pairs should be possible. A first guess is that, as a decision system, the biometric system should be a binary one. Theoretically, in ideal conditions, it should be able to map all its legal inputs (pairs of iris templates) onto a set of two concepts and linguistic labels 'imposter' and 'genuine' whose extensions should be disjoint, since in a logically consistent iris recognition system and also in our reasoning, no imposter 
pair is a genuine one and vice versa. Unfortunately, the field of iris recognition is full of counter-examples to this ideal situation, some of them as old as the domain of iris recognition itself [3], [4] and others, very recent indeed [6]-[10], [24], [25], some of them in more direct connection with what follows to be presented in this paper [2], [12]-[17], [19]-[23]. In all of these counter-examples it happens that the linguistic labels 'imposter' and 'genuine' are, in fact, represented as two overlapping fuzzy sets of recognition scores, the overlapping being itself a fuzzy boundary in between the first two mentioned fuzzy sets. Therefore, a second guess is that, as a decision system, the biometric system outputs three possible values: 'imposter', 'uncertain' and 'genuine'. A step further is analyzing the causes for which some imposter similarity scores are too big and some genuine similarity scores are too small, in an attempt to better isolate from each other the extreme values imposter and genuine. The result is a 5-valued recognition function that splits the input space (of iris template pairs) in five fuzzy preimages of five labels imposter, hyena (degraded imposter), uncertain, goat (degraded genuine) and genuine, all of them represented as fuzzy sets of recognition scores. All in all, the non-ideal conditions occurred from the stage of image acquisition up to the stages of image processing and matching fuzzify the prototype binary crisp recognition function that all biometric systems are normally expected to have (in ideal conditions) up to a binary, ternary, quaternary or even quinary fuzzy recognition function. Therefore, the initial intention of designing an identification system must be weakened when necessary to designing verification systems. However, when the aim is to design a logically consistent recognition system within the limits of consistent biometry [22], the difference between verification and identification vanishes.

This framework of iris recognition (and biometric recognition at large) is the context in which we talk here about fuzzy membership, probability and negation while searching for appropriate ways of expressing (precisiating, [31]) facts, rules and phenomena of iris recognition in a computational manner such that to maximize the cointension [31] between the real world of iris recognition and its computational model. By adopting a Turing perspective [27], we classify such a task as a process of human intelligence and its computational model as an artificial agent whose degree of intelligence can be determined through the test that today bears his name (Turing test, [27]). In other words, ideally, artificial intelligence is a way of representing processes of human intelligence as computational (artificial) software agents with as much cointension as possible, the degree of cointension being verifiable in principle [1] through a Turing test [27]. The results of all major iris recognition experiments (such as [4], [8], [10], [13], [19], [20], [22], [24]) are in fact partial Turing tests of iris recognition in which only the software agent is interrogated. Completing these partial tests can be done easily by attaching to them the corresponding iris recognition results obtained while interrogating qualified human agents using the same iris image databases. Surprisingly, especially when using iris image databases containing good quality images, the histogram of biometric decisions given by the human agents are indeed very 
different by those summarizing the biometric decisions given by the artificial agents (the iris recognition systems). The huge discrepancy between the two types of iris recognition results was for the first time observed and understood in [21] and further commented and used in [22] as an argument for searching a better methodology for non-stationary machine-precisiation of iris recognition. Here we will insist now on the lack of cointension between the human-precisiated and machine-precisiated iris recognition and on the lack of instruments for quantifying it. Let us start with a tentative of quantifying the degree of separation between the distributions of imposter and genuine similarity scores. In the classical statistical approach of iris recognition proposed by Daugman, the decidability index,

$$
d^{\prime}=\frac{\left|\mu_{I}-\mu_{G}\right| \sqrt{2}}{\sqrt{\sigma_{I}^{2}+\sigma_{G}^{2}}},
$$

is such a measure of separation (where $\mu_{I}, \mu_{G}, \sigma_{I}^{2}$ and $\sigma_{G}^{2}$ are the means and the variances of imposter and genuine score distributions respectivelly), values of 4 , 8 , and 14 being already reported in the literature [3], [4]. Hence, the separation between the distribution of imposter and genuine similarity scores is in this case precisiated numerically in such numeric values exemplified above. However, the histograms summarizing the biometric decisions given by qualified human agents are crisp 0-1 (binary) histograms for which the same decidability index takes the value of positive infinity. How relevant are 4,8 , or 14 against the infinity? How much cointension is in this case in between the human-precisiated and machineprecisiated iris recognition? We could not say otherwise than not too much at all, and this is what motivates our paper, which is further organized as follows:

\subsection{Outline}

Section 2 presents a newly proposed formalization of the classical probabilitypossibility relation, whereas the sections 2.1 and 2.2 introduce the notions of consistent experimental setups and frameworks exemplified in section 2.3. Section 3 presents and analyzes some cases of imperfect experimental frameworks (especially cases of iris recognition, see section 3.1) study that leads to the finding of a new possibility - probability - fuzzy membership relation for Gaussian distributed random numbers and also for other continuously distributed random numbers, finding that points out that weakening the $\sigma$-additivity condition is not necessarily required for establishing a consistent possibility-probability-fuzzy membership relation. On the contrary, the $\sigma$-additivity condition is the bridge that ensures this relation and therefore statistics and fuzzy logic could share a common side-by-side evolution - fact that is commented in section 3.4. The sections 3.5 and 3.6 are dedicated to exemplifying some issues that negation has when it comes to deal with fuzzy membership assignments. At last, the $4^{\text {th }}$ section deals with two types of negation in the context of implemented biometric systems and is followed by conclusions. 


\section{A new formalization of the classical probability- possibility relation.}

Let us consider a data spring, i.e. an input-output relation $\mathrm{R}$ of a theoretical system $\mathrm{S}_{\mathrm{T}}$ actually implemented as $\mathrm{S}_{\mathrm{P}}$, a data spring that throws an uniformly distributed number $\mathrm{y}$ in the discrete set of fuzzy values $\mathrm{Y}=[0: 1: 255] / 255=\left\{\mathrm{y}_{\mathrm{k}}=\mathrm{k} / 255\right.$ | $\left.\mathrm{k} \in \mathrm{Z}_{256}\right\}$ as a response to the excitation $\mathrm{x} \in \mathrm{X}$. By intentionally confounding the output $\mathrm{y}$ with the state of system $\mathrm{S}, \mathrm{X}$ is the input space and $\mathrm{Y}$ is the state-output space. Given the fact that the data spring is a uniformly distributed number in $\mathrm{Y}$, all $\mathrm{y} \in \mathrm{Y}$ are observable outputs/states and the event $\mathrm{y} \in \mathrm{Y}$ is not just possible, but certain. The possibility that $y \in Y$ originates in the nature of data spring $R$, whereas for any $R$ taken such that $R(X)=Y$, the probability of $y \in Y$ is unitary. In such cases, while the system $S_{P}$ is functioning, the actual outcome $y=R(x)$ cannot enter in the output/state space through its empty subset, i.e. the event $R(x) \notin Y$ is impossible, whereas its probability is zero. In such cases, even if we consider the possibility is a matter of degree, the (maximally) impossible event is not improbable (as Zadeh said in [30]), but actually it is not probable at all (i.e. it is 0 -probable). On the other hand, the maximally possible event is the certain event whose probability is unitary. In what follows here, all 0 -probable events are impossible, all p-probable events with $1 \geq p>0$ are possible and all 1-probable events are not just possible, but certain. The possibility of an event is not fuzzy, but binary: all possible or impossible events have their possibility coefficients equal to 1 or 0 , respectively. Hence probability follows, originates in and expresses possibility (probable events are not impossible) and possibility is causal to probability. This state of facts is already expressed in probability theory as a precise law, namely the $\sigma$-additivity axiom within the definition of probability, which can be also viewed as a principle of consistent possibility-to-probability translation, i.e. as an instance of the so called possibility/probability consistency principle introduced by Zadeh in [30],

$\gamma=\sum_{k=1}^{n} p_{k} \pi_{k}$, with $p$ and $\pi$ denoting probability and possibility values,

where all possibility values are taken unitary. In our modeling, a state or an output is observable if and only if it is possible, i.e. the possibility and observability are interchangeable (synonyms). In terms of formal languages, there is a formal grammar that describes the systems $S_{T}$ and $S_{P}$ able of producing that specific state/output also. In a consistent experimental setup, all possible events should be observed and, as a consequence, their statistics can be made, probability being a nuance, a refinement of possibility (among all possible events, some are more probable than others), a finer precisiation / quantization of possibility in a numeric space after the knowledge resulting from a certain experiment is gathered as statistics data. Hence, the main differences between the classical possibilityprobability relation that we are bounded to here and the model proposed by Zadeh in [30] are the following: 


\begin{tabular}{|c|c|}
\hline Possibility is encoded binary. & Possibility is a matter of degree. \\
\hline $\begin{array}{c}\text { Possibility and probability values } \\
\text { satisfy the } \sigma \text {-additivity condition, } \\
\text { and therefore, they are cointensive } \\
\text { and fully consistent to each other- } \\
\text { i.e. in formula (2), } \gamma \text { and possibility } \\
\text { values are all unitary. }\end{array}$ & $\begin{array}{l}\text { Possibility and probability values are } \\
\text { not necessarily cointensive, nor fully } \\
\text { consistent to each other, since their } \\
\text { degree of consistency (2) marks a } \\
\text { weakening of the } \\
\sigma \text {-additivity condition. }\end{array}$ \\
\hline \multicolumn{2}{|c|}{ For any consistent experimental setup 'E', any event 'e' and any $p \in(0,1]$ : } \\
\hline impossible $(e) \leftrightarrow 0$-probable $(e, E)$ & impossible $(\mathrm{e}) \rightarrow$ improbable(e) \\
\hline possible $(\mathrm{e}) \leftrightarrow$ p-probable $(\mathrm{e}, \mathrm{E})$ & $\begin{array}{c}\text { probable }(\mathrm{e}) \rightarrow \text { possible }(\mathrm{e}) \\
\text { (if contrapositive principle still stands) }\end{array}$ \\
\hline certain $(\mathrm{e}) \leftrightarrow 1$-probable $(\mathrm{e}, \mathrm{E})$ & formula (2) \\
\hline
\end{tabular}

Let us comment now on these matters. First of all, the relation between impossibility and 0-probability,

impossible(e) $\leftrightarrow$ 0-probable(e, E),

tells that in any consistent experimental setup ' $E$ ' (situation further denoted as $\mathrm{E} \in \xi$, where the space of consistent experimental setups $\xi$ follows to be defined on the run, by natural restrictions that appear during formalization), an impossible event cannot be observed as an outcome, or otherwise, the experimental setup is not consistent (situation further denoted as $\mathrm{E} \notin \xi$ ). This mechanism can be used to endow any computational artificial agent with the capacity of predicting (having an expectation and a prior knowledge on) the future outcomes of an experiment that it follows to witness, observe and understand, and also with the capacity of knowing who is responsible when these outcomes do not meet its expectation. However, formula (3) is a simplified instance of a more complex one that belongs in a second-order formal language describing the systems $\mathrm{S}_{\mathrm{T}}$ and $\mathrm{S}_{\mathrm{P}}$ (in what follows, $\mathrm{t}$ and $\mathrm{f}$ are used as true and false):

impossible $(\mathrm{e}) \leftrightarrow\{(\forall \mathrm{E} \in \xi)[\mathrm{t} \rightarrow 0$-probable $(\mathrm{e}, \mathrm{E})]\}$

telling that an event ' $\mathrm{e}$ ' is impossible if and only if, in any consistent experimental setup ' $E$ ', its probability is null. Its dual by contraposition principle is:

possible $(\mathrm{e}) \leftrightarrow\{(\exists \mathrm{E} \in \xi)[0$-probable $(\mathrm{e}, \mathrm{E}) \rightarrow \mathrm{f}]\}$

telling that an event ' $\mathrm{e}$ ' is possible if and only if there is a consistent experimental setup in which the assertion that ' $\mathrm{e}$ ' is 0 -probable in ' $\mathrm{E}$ ' is false. Formula (5) is further equivalent to:

possible $(\mathrm{e}) \leftrightarrow\{(\exists \mathrm{E} \in \xi)(\exists \mathrm{p} \in(0,1])[\mathrm{t} \rightarrow \mathrm{p}$-probable $(\mathrm{e}, \mathrm{E})]\}$

telling us that an event ' $\mathrm{e}$ ' is possible if and only if there is a consistent experimental setup in which the assertion that the probability of ' $\mathrm{e}$ ' is not null in ' $\mathrm{E}$ ' is true.

Secondly, the relation between possibility and p-probability (when $p \in(0,1]$ ), 
possible $(\mathrm{e}) \leftrightarrow$ p-probable $(\mathrm{e}, \mathrm{E})$,

is also a simplified instance of a more complex formula:

possible $(\mathrm{e}) \leftrightarrow\{(\forall \mathrm{E} \in \xi)(\exists \mathrm{p} \in(0,1])[\mathrm{t} \rightarrow$ p-probable $(\mathrm{e}, \mathrm{E})]\}$,

that tells that an event ' $\mathrm{e}$ ' is possible if and only if, in any consistent experimental setup ' $E$ ', (is true that) its probability is not null. Formula (8) is actually a strengthening of (6) by universal quantification of $\mathrm{E}$ in $\xi$, and therefore, a strengthening of (4). Besides, the relations (8) and (5) could not be simultaneously true outside $\xi$, hence $\xi$ is necessarily defined as follows:

\subsection{Consistent experimental setups}

Definition 1 The space of consistent experimental setups $\xi$ for the system $\mathrm{S}_{\mathrm{T}}$ whose observable state/output space $\mathrm{Y}$ is entirely covered by its input-output $(\mathrm{X}-\mathrm{R}-\mathrm{Y}$ ) relation (i.e. $\mathrm{R}(\mathrm{X})=\mathrm{Y}$ ) is a space of experiments (on implemented systems $\mathrm{S}_{\mathrm{P}}$ ) in which:

(i) any two members are interchangeable, i.e.:

$\mathrm{t} \rightarrow\{[(\exists \mathrm{E} \in \xi)(\mathrm{P}(\mathrm{e}, \mathrm{E}))] \rightarrow[(\forall \mathrm{E} \in \xi)(\mathrm{P}(\mathrm{e}, \mathrm{E}))]\}$,

where $\mathrm{P}(\mathrm{e}, \mathrm{E})$ is a property of ' $\mathrm{E}$ ' relative to a given event ' $\mathrm{e}$ '.

(ii) the following rewriting rule holds true:

$\{(\exists \mathrm{p} \in(0,1])[\mathrm{t} \rightarrow$ p-probable $(\mathrm{e}, \mathrm{E})]\} \leftrightarrow[0$-probable $(\mathrm{e}, \mathrm{E}) \rightarrow \mathrm{f}]$

(iii) there is a negation operator ' $n$ ' defined such that $n^{2}=1$ (in terms of string functions) and:

$\mathrm{n}\{(\forall \mathrm{E} \in \xi)[\mathrm{t} \rightarrow 0$-probable $(\mathrm{e}, \mathrm{E})]\} \leftrightarrow\{(\exists \mathrm{E} \in \xi)[0$-probable $(\mathrm{e}, \mathrm{E}) \rightarrow \mathrm{f}]\}$,

(iv) the following rewriting rule for complementary events e and $\overline{\mathrm{e}}$ holds true:

$(\forall \mathrm{E} \in \xi)(\forall \mathrm{p} \in[0,1])\{[\mathrm{p}-\operatorname{probable}(\mathrm{e}, \mathrm{E})] \leftrightarrow[(1-\mathrm{p})-\operatorname{probable}(\overline{\mathrm{e}}, \mathrm{E})]\}$

$(8) \leftrightarrow(4)$

$\uparrow$

$\frac{(8) \leftrightarrow(5)}{\uparrow} \quad$ AND $\quad(5) \leftrightarrow(4)^{*}$

$(8) \leftrightarrow(6) \quad$ AND $\quad(10)$

$\uparrow$

(where * is provable by (11) and contraposition principle)

In these conditions, a formal proof that $(8) \leftrightarrow(4)$ is presented here as formula (13), where the equivalence relation * within the formula (13) is provable by (11) 
and contraposition principle. The condition (iv) within Definition 1 establishes the natural meaning of both labels impossible and certain by correspondence with the trivial two-elements Boolean algebra defined by the empty set and Y, on the one hand, and with the two extreme values ( 0 and 1$)$ that the cumulative of probability takes for the empty (impossible) and total (certain) events, respectively, on the other.

In the third place, the relation between certain and 1-probable events:

certain(e) $\leftrightarrow$ 1-probable(e, E),

is also a simplified instance of a more complex formula:

certain $(\mathrm{e}) \leftrightarrow\{(\forall \mathrm{E} \in \xi)[\mathrm{t} \rightarrow 1$-probable $(\mathrm{e}, \mathrm{E})]\}$,

affirming that the event ' $e$ ' is certain, if and only if, it is 1-probable in any consistent experimental setup ' $E$ '. Given the rewriting rule (iv) stated in Definition 1, formula (15) is further equivalent to:

impossible $(\overline{\mathrm{e}}) \leftrightarrow\{(\forall \mathrm{E} \in \xi)[\mathrm{t} \rightarrow 0$-probable $(\overline{\mathrm{e}}, \mathrm{E})]\}$,

that further is an instance of formula (4). By summarizing this section up to this point, an important remark is that the three ways of describing the classical possibility-probability relation for the impossible, possible and certain events, namely the formulae (4), (8) and (15), or their simplified forms (3), (7) and (14) and also the axioms within the definition of probability are not independent, but intimately interconnected as three images of the same thing, namely the concept denoted above as $\xi$ - the space of all consistent experimental setups $\xi$ for the system $\mathrm{S}_{\mathrm{T}}$ whose observable state/output space $\mathrm{Y}$ is entirely covered by its inputoutput relation. $\xi$ is a formal, logical, computational and physical concept, a coherent non-contradictory framework of expressing the natural relation between possibility, probability and negation for all observable states/outputs of a physical system $\mathrm{S}_{\mathrm{P}}$ given as an implementation of $\mathrm{S}_{\mathrm{T}}$. By contrast, now the reader knows what could mean to weaken any of the three axioms within the probability definition while attempting to define a possibility-probability relation different from the canonic natural one that exists by default in $\xi$.

A second remark is that investigating the possibility-probability relation in $\xi$ shows how many things confirm each other and group together coherently in a consistent and computational knowledge ensemble.

A third remark is that all formulae from above that contain the symbols $\mathrm{t}$ and $\mathrm{f}$ i.e. (4)-(6), (8)-(12), (15) and (16) - are written in a formal logical dialect that extends the cognitive dialect introduced in [17], which on its turn is derived from the sound and complete formal theory of Computational Cognitive Binary Logic (CCBL) introduced in [15] and implemented online in [18]. However, the marker (!:) signalizing an assertion in cognitive dialect is omitted in the above formulae, because here in this article there is no chance of confusing queries and assertions. Hence, our discourse presented here on the possibility-probability relation is strictly a logical and computational one. 


\subsection{Consistent experimental frameworks}

An artificial computational agent produces its states/outputs accordingly to a sound formal theory in which the states/outputs are formally and logically provable in terms of a generative grammar. Hence, in $\xi$, a more complex relation holds between possibility, probability, observability and provability: observable events (states/outputs) of $\mathrm{S}_{\mathrm{T}}$ and $\mathrm{S}_{\mathrm{P}}$ are possible, probable and provable, any time when the input-state/output relation is theoretically known as a provable formula of a sound theory describing $\mathrm{S}_{\mathrm{T}}$ and practically implemented in $\mathrm{S}_{\mathrm{P}}$ without errors. This motivates the following definition:

Definition 2 A Consistent Experimental Framework (CEF) is a formal ensemble $\left(\mathrm{X}, \mathrm{R}, \mathrm{Y}, \mathrm{S}_{\mathrm{T}}, \mathrm{T}_{\mathrm{S}}, \xi\right)$ in which:

(i) $\xi$ is the space of consistent experimental setups for the system $\mathrm{S}_{\mathrm{T}}$;

(ii) the observable state/output space $\mathrm{Y}$ of the system $\mathrm{S}_{\mathrm{T}}$ is entirely covered by the input-output (X-R-Y) relation (i.e. $\mathrm{R}(\mathrm{X})=\mathrm{Y})$;

(iii) input-state/output relation (X-R-Y) is know explicitly as a provable formula of a sound formal theory $\mathrm{T}_{\mathrm{S}}$ describing $\mathrm{S}_{\mathrm{T}}$, on the one hand, and implemented without errors on $\mathrm{S}_{\mathrm{P}}$, on the other.

In this context, since $\mathrm{T}_{\mathrm{S}}$ is a sound formal theory, it cannot prove contradictions. Therefore, contradictions are impossible / unreachable / unprovable events in $T_{S}$. Since the formal theory of $T_{S}$ actually describes the functioning of $S_{T}$ and $S_{P}$, the practice on $S_{\mathrm{P}}$ is not able to deliver events that are theoretically impossible / unreachable / unprovable (in $\mathrm{S}_{\mathrm{T}}$ ), and therefore, the practice on $\mathrm{S}_{\mathrm{P}}$ (further denoted $\mathrm{P}_{\mathrm{S}}$ ) cannot deliver counter-examples for $\mathrm{S}_{\mathrm{T}}$ and $\mathrm{T}_{\mathrm{S}}$. In other words, there is a unitary cointension between $S_{T}$ and $S_{P}$, between the theory $T_{S}$ and the practice $P_{S}$ on the theoretical and actual systems $\mathrm{S}_{\mathrm{T}}$ and $\mathrm{S}_{\mathrm{P}}$. In general, the lack of cointension between practice (experiments) and theory could be expressed in experimental results that contradict the theoretical model of the system in some aspects. On the contrary, a unitary cointension between practice and theory ensures that in any consistent experimental setup it is impossible to obtain experimental results that contradict the expectations motivated by the sound theory $\mathrm{T}_{\mathrm{S}}$. There are only two possible gates that could allow inconsistency (contradiction) within an experimental framework, namely $\mathrm{T}_{\mathrm{S}}$ is not sound or $\mathrm{P}_{\mathrm{S}}$ is outside $\xi$. In addition, there are three ways in which contradiction could be expressed in an experimental framework: inside $T_{S}$, inside $P_{S}$, and between theoretically predicted events in $T_{S}$ and the actual events taking place in $P_{S}$, i.e. between expectations and the actual experimental outcomes.

\subsection{Examples of consistent experimental frameworks}

The extension of the concept introduced in Definition 2 from above is not the empty set: propositional logic is the formal theory that describes any logical circuit both as theoretical design $\mathrm{S}_{\mathrm{T}}$ and as implemented system $\mathrm{S}_{\mathrm{P}}$. Given a 
sequence of excitations in the input space, the corresponding outputs are formally provable in propositional logic (hence, accordingly to the formal theory of the system they are expected values) and practically observable. For a given inputoutput relation, any two consistent experimental frameworks differ only by equivalent (interchangeable) logical circuits that support that input-output relation. Given an input-output relation and a trajectory $(\mathrm{x}, \mathrm{y})_{\mathrm{t}}$ in $\mathrm{X} \times \mathrm{Y}$, output statistics is invariant along all consistent experimental frameworks that support that inputoutput relation, and therefore, the possibility-probability relation is invariant.

This example is also important for establishing two things, once for good: logical circuits are the most basic intelligent agents (where intelligence means Turing defined intelligence, i.e. the artificial intelligence provable by Turing tests) and secondly, in a Turing test, it is mandatory that the human agent to be qualified (besides being informed). Obviously, there is only one chance for the human agent to predict correctly all outputs of a logical circuit, namely to know propositional logic (besides knowing the circuit design). Otherwise, the results of a Turing test on a logical circuit are not relevant at all. On the other hand, the human or artificial agent that organizes the Turing test must be able to recognize intelligence regardless if is human intelligence or artificial intelligence. The true output is either certain and 1-probable or possible and p-probable (with $p \in(0,1)$ ) or impossible and 0-probable for any logical circuit implementing a tautology / a contextual truth / a contradiction, respectively.

A second example of consistent experimental frameworks can be built for any digital circuit in general, by analogy with the first example from above. We make this remark because, ultimately, a biometric system can be viewed today as a complex digital circuit.

\section{Imperfect experimental frameworks}

$\xi$ and CEF are introduced exactly for ensuring that what is theoretically possible / probable / certain is also practically possible / probable / certain, respectively. On the other hand, $\xi$ and CEF allow us to study weakened models, when the weakening is made otherwise than changing the axioms within the definition of probability, for example. We do not have any clue that weakening probability axioms could prove to be maximally productive, because even in imperfect experimental frameworks the probability theory continues to function despite the adequacy of our beliefs and intentions. In other words, when a system evolves on and within measurable sets/spaces, we can ignore the probability theory if we prefer, but we cannot abolish it, simply because it is engraved/embedded within the structure of the space itself. Besides, we will illustrate further, how probability distribution functions (PDFs) and cumulative distribution functions (CDFs) appearing in biometrics (or anywhere else) can generate fuzzy membership functions easily, in more than a single way. Hence, adopting a fuzzy approach in a 
given matter is not necessary equivalent with contradicting probability theory (by weakening some of its axioms). The major difference between our approach and [30] is that in ours, the classical possibility-probability relation (described above in terms of $\xi$ and $\mathrm{CEF}$ ) stays unchanged while the experimental frameworks that we work with - despite being allowed to be imperfect - are not allowed to decay up to the abolition of probability theory.

A first degree of imperfection illustrated here is when accurately implementing $\mathrm{S}_{\mathrm{T}}$ is practically impossible or technically and economically unfeasible. For example, when $\mathrm{N}$ is big enough, a preferable alternative for implementing $\mathrm{N}$-dimensional dynamical systems with predefined input-output behavior is designing a simpler n-dimensional system $(\mathrm{n}<<\mathrm{N})$ that supports almost the same input-output behavior - a technique known as order reduction. However, in such case, the practical $\mathrm{n}$-dimensional implementation $\mathrm{S}_{\mathrm{Pn}}$ and the theoretical $\mathrm{N}$-dimensional model $\mathrm{S}_{\mathrm{TN}}$ are still highly cointensive, whereas the practical n-dimensional implementation $\mathrm{S}_{\mathrm{Pn}}$ and the theoretical $\mathrm{n}$-dimensional reduced model $\mathrm{S}_{\mathrm{Tn}}$ are still totally cointensive, and therefore, the classical possibility-probability relations (3), (4), (7), (8), (14) and (15) still hold true in the consistent experimental framework $\left(\mathrm{X}, \mathrm{R}, \mathrm{Y}, \mathrm{S}_{\mathrm{Tn}}, \xi\right)$ when $\xi$ is $\mathrm{S}_{\mathrm{Pn}}$ based.

\subsection{Imperfect experimental frameworks in iris recognition}

One of the worst but still manageable (hence acceptable) degree of imperfection is when the required (target) input-output (i-o) behavior is known as being possible, but all practical implementations $S_{P}$ attempted up to some point for mimicking the target $\mathrm{i}$-o behavior are almost failed resulting in practical $\mathrm{i}-\mathrm{o}$ behaviors that are very different from the target i-o behavior. This is currently the case of all iris recognition results and biometric systems belonging to the statistical paradigm pioneered by Daugman [3], [4]. The target i-o behavior assigned to an a priori unknown target system $\mathrm{S}_{\mathrm{T}}$ that the user intends to design (proved possible by interrogating a qualified human agent during a Turing test while using good quality eye images) can be statistically illustrated as a 0-1 histogram of correct biometric decisions ( 0 - for identifying a pair of iris images taken for different eyes, 1 - for identifying a pair of iris images taken for the same eye), as in Figure 1, whereas the i-o behavior of implemented system is statistically illustrated in Figure 2. As exemplified in Figure 1 and Figure 2, plotting the statistics of the results recorded in a Turing test is a way of visually quantifying the loss in precision occurred for the implemented system in comparison with the target i-o behavior. The lack of cointension between target i-o behavior and implemented i-o behavior is obvious. Figure 1 illustrates a logically consistent, crisp, artificial and binary understanding (logical, crisp, binary and lossless human-precisiation of meaning) for two concepts whose extension are not just disjoint but complementary in the input space of the recognition system: 'imposter pairs' (IP) and 'genuine pairs' (GP). Figure 2 illustrates a lossy compression of the original meaning of the two concepts, a fuzzy artificial perception and a fuzzy 
machine-precisiation of meaning for the two original human-precisiated concepts. The artificially perceived fuzzy concepts (further denoted as f-IP and f-GP or as fimposter and f-genuine) are no longer disjoint. There are pairs of irides qualifying simultaneously and equally as f-genuine and f-imposter pairs, while others qualify ambiguously but with different probabilities. A better situation in terms of cointension can be observed by comparing Figure 1 from here to Figure 2 from [19], Figure 4 from [20] and Figure 5 from [22].

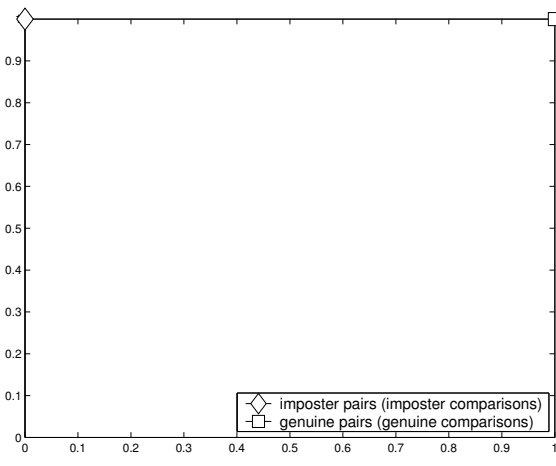

Figure 1

Human-precisiation of iris recognition: PDFs corresponding to the correct biometric decisions given by a qualified human agent during a Turing test of iris recognition (diamond for imposter pairs, square for genuine pairs)

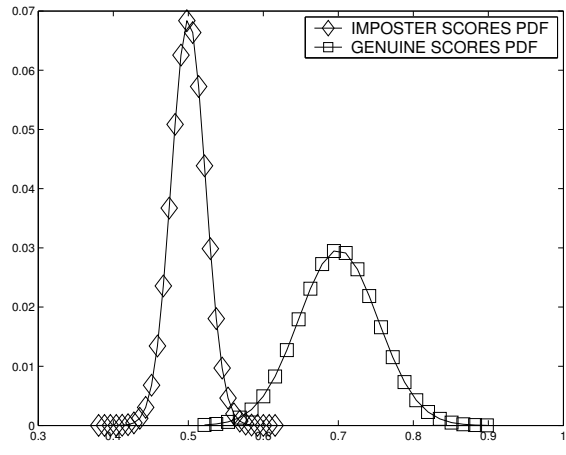

Figure 2

Machine-precisiation of iris recognition: PDFs of the imposter scores (diamond) and genuine scores (square) collected during a Turing test of iris recognition while interrogating an artificial agent implementig statistical iris recognition.

The imperfection of such an experimental framework illustrated in Figure 1 and Figure 2 is further commented in terms of possibility-probability relation: in theoretical target system corresponding to Figure 1 the confusion between an imposter and a genuine pair is impossible, whereas in the implemented system it is possible, indeed. Forcing the correct recognition of all imposter pairs within the implemented system comes at the price of not recognizing correctly all genuine pairs. This trade-off is inherent in classical statistical iris recognition. Therefore, despite the original concepts consistently support negation, the fuzzy concepts f-IP and f-GP cannot be consistently negated.

The consistent experimental frameworks are contexts in which the cointension between a target formal theory and the actual implemented system transports probability-possibility relation between theory and practice in the same manner in which continuity transports convergence from the argument space to the image space in the framework of topology. On the contrary, in the imperfect experimental frameworks the cointension between theory and practice (or between target and implemented system) can be so weak that even theoretically but still logically impossible events could appear as practically probable (hence practically possible). However, the fact that cointension is weak enough and consequently unable to establish a consistent bridge between what is practically probable and theoretically possible does not mean that the possibility-probability relation is 
broken inside the set of events concerning the implemented system alone: confusion between the two artificially perceived concepts (imposter and genuine pairs) is practically p-probable $(1>p>0)$ and therefore practically possible. On the other hand, when logical inconsistency gets its place inside an imperfect experimental framework, the right contra-measure to take is attempting to recover logical consistency, not attempting to redefine possibility-probability relation, because otherwise, contradiction being explosive, sooner or later, anything can be proved and some logically impossible events could appear as proved/supported by "experimental evidence" to be probable (hence possible) events. In short, when it comes to test or to design systems in imperfect experimental frameworks, there is no sound argument to extrapolate experimental evidence to expectations for real life without precautions, regardless if the channel on which the extrapolation is made is possibility-to-probability or possibility-to-fuzzy membership relation.

\subsection{A possibility - probability - fuzzy membership relation for Gaussian events}

The most important aspect revealed by the comparison between Figure 1 and Figure 2 is the lack of cointension between what is maximally achievable (Figure 1) and what is currently achieved in implementation (Figure 2). The relation between what is theoretically possible and practically probable is broken, whereas the relation between what is practically possible and practically probable still stays consistent. Translating probability to fuzzy membership is possible in many ways. However, this operation is closed in the semantics of implemented system, is an operation whose result is at most an equivalent of some facts already known, i.e. it is at most a rule for rewriting known facts, not an attempt to improve the actual implemented system. Regardless the way chosen to express fuzzy membership based on statistics of experimental data, in the absence of cointension, there is no instrument to carry this information back and forth between theory and practice. Besides, as the following example illustrates, forcing the meaning of the data to conform to a pattern that it is not really exhibited as an observable behavior, inevitably brings more inconsistency to an already imperfect experimental framework. In short, interpreting the experimental data does not solve the problems, but only point out to them.

Since in the case investigated here, the errors are inherent to the implemented system (Figure 2), computing right-to-left CDF under the imposter distribution (FAR - False Accept Rate, diamond markers in Figure 3 and Figure 4) and left-toright CDF under the genuine distribution (FRR - False Reject Rate, square markers in Figure 3 and Figure 4) define a way of interpreting fuzzy membership of the recognition scores obtained by the input iris pairs to two fuzzy sets corresponding to the two artificially perceived concepts f-imposter and f-genuine scores (see Figure 3 and Figure 4). However, according to this interpretation, 0 is an imposter score (a convenable interpretation, given the target behavior in Figure 1), fact that is neither confirmed experimentally (Figure 2) nor confirmed 
theoretically in statistical iris recognition. Moreover, the means of the two Gaussian variables in Figure 2 have the degrees of membership to the fuzzy sets fimposter and f-genuine scores expressed as 0.5 , which is clearly counter-intuitive, the mean being the most representative sample of a Gaussian signal.

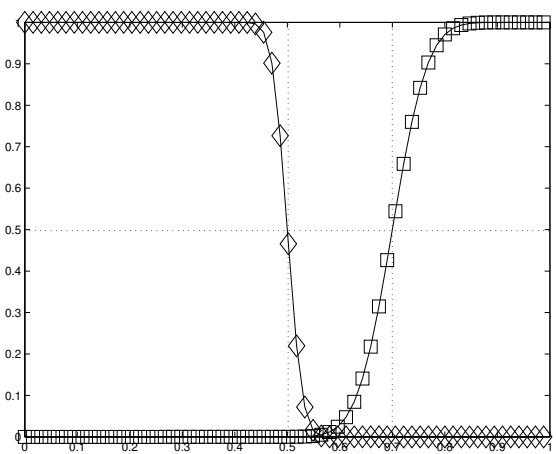

Figure 3

Machine-precisiation of statistical iris recognition: False Accept Rate (FAR, diamond markers) and False Reject Rate (square markers) represented with liniear y coordinate; zoom to membership degrees that the means of the two Gaussians (Figure 2) have in f-imposter and f-genuine fuzzy sets.

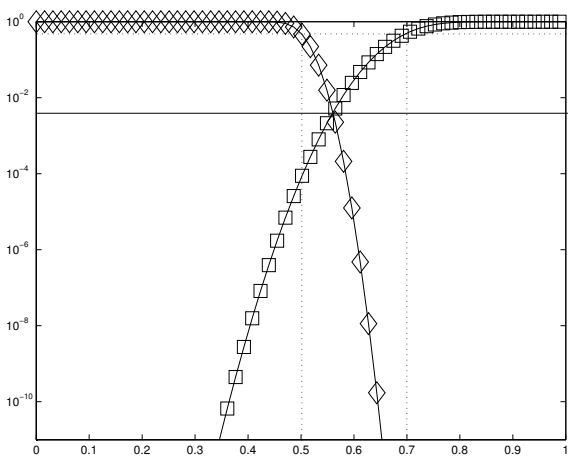

Figure 4

Machine-precisiation of statistical iris recognition: False Accept Rate (FAR, diamond markers) and False Reject Rate (square markers) represented with logarithmic y coordinate - zoom to the Equal Error Point (indicated above by the continuous horizontal line situated slightly under 1E-2).

Experimental data can be interpreted using fuzzy if-then rules. For example, the ensemble of fuzzy if-then rules producing the fuzzy-membership assignments represented in Figure 3 as FAR and FRR curves is the following:

- fewer successors a score has within the imposter distribution, weaker its degree of membership to f-imposter fuzzy set is;

- fewer predecessors a score has within the genuine distribution, weaker its degree of membership to f-genuine fuzzy set is;

However, the same interpretation can easily fail in contradiction or in counterintuitive facts, as illustrated above.

A different interpretation of experimental data can be made accordingly to the following ensemble of fuzzy if-then rules, which is better suited for describing Figure 2 (probability) in terms of fuzzy membership as in Figure 5:

- for scores situated on the right/left side of the mean, fewer successors / predecessors a score has within the (imposter or genuine) distribution, weaker its degree of membership to the corresponding fuzzy set (f-imposter or fgenuine) is;

- recognition score equals to the mean of (imposter or genuine) distribution has unitary membership degree with respect to the corresponding fuzzy set (fimposter or f-genuine); 


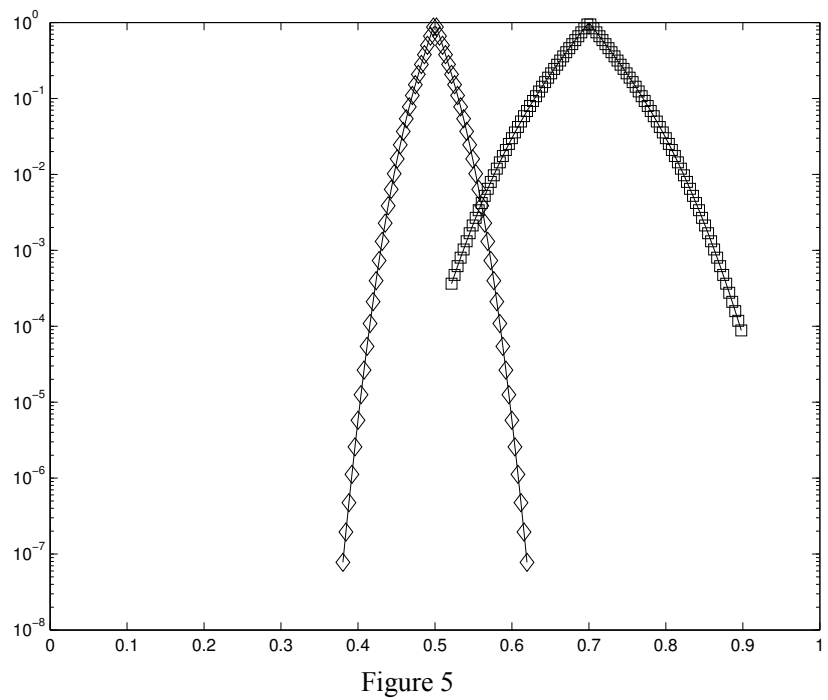

Machine-precisiation of statistical iris recognition: fuzzy membership assignment of the recognition scores obtained experimentally and represented in Figure 2 as the two fuzzy sets fimposter (diamond markers) and to f-genuine (square markers).

Given the symmetry of Gaussian variables, the fuzzy membership function should also be symmetric. For any given distribution and any given score from Figure 2 there is a pair of left- and right-side cumulatives under that distribution and the sub-unitary ratio of these two cumulatives is a plausible fuzzy membership assignment of the given score to the corresponding fuzzy set (f-imposter or fgenuine) that satisfies the two fuzzy if-then rules from above. Fuzzy membership functions constructed this way are illustrated in Figure 5, which represents a fuzzy decisional model associated to the statistics of experimental data within Figure 2. This second interpretation of the experimental data within Figure 2 is better that the previous one (illustrated in Figure 3 and Figure 4) at least for three reasons: the means are scored with a unitary degree of membership, membership is not arbitrarily extended to scores that are not obtained experimentally, and at last, but not the least, the abscise of the Equal Error Point is preserved from Figure 4 to Figure 5 (i.e. the point of equal error expressed in Figure 5 in terms of fuzzy memberships corresponds exactly to the point of equal error expressed in Figure 4 in statistical terms of FAR and FRR).

Summarizing, even in imperfect experimental frameworks, iris recognition experimental results can be expressed in terms of practical possibility - probability - fuzzy membership on the experimental side of the framework, because a recognition event ' $e_{s}$ ' with a given score ' $s$ ' recorded experimentally (in Figure 2) is practically possible, practically probable and maps the input pair into the two fuzzy sets f-imposter through the fuzzy membership assignment illustrated in Figure 5. However, in an imperfect experimental framework there are at least two unsolved problems: 
- the formal logic involving experimentally recorded recognition events is contextual and temporal because all true sentences that can be derived on the experimental side of the framework are true at some specific time, in some specific case, accordingly to a specific experiment, accordingly to specific experimental data. This situation emphasizes the importance of condition (9) within the Definition 1.

- the huge difference between what is achievable (in human-precisiated iris recognition Figure 1) and what is achieved (in machine-precisiated statistical iris recognition, Figure 2, Figure 5), a gap that can be filled through creativity only, not through interpretation.

On the other hand, Gaussian events that vanish at some left- and right-side points (for example, the recognition of imposter pairs in statistical iris recognition and the corresponding Gaussian distributed numbers) define specific fuzzy sets as follows: if $\mathrm{s}_{\mathrm{g}} / \mathrm{e}_{\mathrm{g}}$ is a Gaussian distributed number / event vanishing at some left and right points, its membership to any continuous or (discretized) interval considered within the vanishing left and right limits can be expressed as a fuzzy degree of membership (Figure 2, Figure 5):

$\mathrm{FM}\left(\mathrm{s}_{\mathrm{g}}\right)=\min \left(\mathrm{L}_{\mathrm{C}}\left(\mathrm{s}_{\mathrm{g}}\right) / \mathrm{R}_{\mathrm{C}}\left(\mathrm{s}_{\mathrm{g}}\right), \mathrm{R}_{\mathrm{C}}\left(\mathrm{s}_{\mathrm{g}}\right) / \mathrm{L}_{\mathrm{C}}\left(\mathrm{s}_{\mathrm{g}}\right)\right)$,

where $\mathrm{L}_{\mathrm{C}}\left(\mathrm{s}_{\mathrm{g}}\right)$ and $\mathrm{R}_{\mathrm{C}}\left(\mathrm{s}_{\mathrm{g}}\right)$ are the left- and right-side cumulatives under the given Gaussian distribution to the left and right vanishing points, respectively.

\subsection{Natural fuzzification of a continuous distribution}

Theorem 1: For any 1-dimensional, real-valued random variable $\mathrm{s}$ that is continuously distributed on a real interval and vanishes at some left and right points, the fuzzy membership FM defined in formula (17) is bounded in $[0,1]$ and has a single global maximum point $\mathrm{s}_{\mathrm{M}}$ for which $\mathrm{FM}\left(\mathrm{s}_{\mathrm{M}}\right)=1$;

Proof: When thinking at the upper bound of FM, the most favorable situation is for that point $\mathrm{s}_{\mathrm{M}}$ where the right-side cumulative equals the left-side cumulative. Indeed, if such point $\mathrm{s}_{\mathrm{M}}$ exists, $\mathrm{FM}\left(\mathrm{s}_{\mathrm{M}}\right)=1$. Since the left-side cumulative on the given continuous distribution computed in the current point $\mathrm{s}$ with respect to the left vanishing point increases with $\mathrm{s}$ and since the value of the left-side cumulative evolves continuously between 0 and 1 , the point $\mathrm{s}_{\mathrm{M}}$ exists and it is defined by the abscise where the left-side and right-side cumulatives are equal to 0.5 . Hence, FM has upper bound that is also its maximum value 1. Given the definition (17), FM strictly increases with $\mathrm{s}$ - when $\mathrm{s}$ is between left-side vanishing point and $\mathrm{s}_{\mathrm{M}}$, and strictly decreases with $\mathrm{s}$ - when $\mathrm{s}$ is between $\mathrm{s}_{\mathrm{M}}$ and the right-side vanishing point of the given distribution, hence, $\left(s_{\mathrm{M}}, 1\right)$ is the only local and global maximum point of FM, whereas its lower bound is given by the values of FM in the left and right vanishing points of the distribution, namely zero.

Formula (17) establishes the degree of fuzzy membership (FM) as being the most pessimistic degree of interiority that $\mathrm{s}_{\mathrm{g}}$ has with respect to the left and right 
vanishing points of the distribution. FM is bounded between 0 and 1 , and has always a single maximum point. In the case of Gaussian events, this maximum point corresponds to the mean and is the first point around which long enough experiments throws enough neighboring values such that to emulate topological density in a given precision (a property that does not hold true for bimodal Gaussian distributed numbers - for example).

Searching for the right context in which the relation between possibility, probability and fuzzy membership can be consistently expressed for the particular case of experimentally obtained iris recognition results brought us inevitably to a natural (canonic) and universal fuzzification procedure available for an entire class of continuous distributions, for which the formula (17) is formally correct (is actually making sense) due to Riemann integrability of all continuous distributions. Formula (17) is a meeting point where classical logic, modal logic, fuzzy logic, probability theory, measure theory, system theory and topology shake their hands explaining consistently the complex but natural provability observability / reachability - possibility - probability - fuzzy membership integrability relation without pointing out to a need for weakening the $\sigma$-additivity condition within the definition of probability up to the formula (2). The $\sigma$-additivity condition is actually the true principle of possibility-probability consistency. Conversely, not any fuzzy membership assignment that we would wish to operate with can be consistently mapped onto a continuous distribution, especially when it is not compatible with the $\sigma$-additivity condition and consequently, the interpretation given by such fuzzy membership assignments can be neither confirmed nor infirmed by measurable experiments (organized in consistent experimental frameworks). Therefore, the reader should figure out if using such fuzzy membership assignments in connection with measurable things and spaces is a matter of excessive oratorical talent or a matter of logic and sound science.

\subsection{The future of statistics and fuzzy logic}

In the context of measurable spaces and consistent experimental frameworks, the $\sigma$-additivity condition ensures that the answer to the question "is there a need of fuzzy logic?" (Zadeh, [31]) is "yes, in the same degree in which there is a need for statistics, classical logic, modal logic, system theory, measure theory and topology". Otherwise, operating fuzzy logic while weakening $\sigma$-additivity condition results in a contradiction, namely the finding of a hypothetically consistent way (science/theory) of quantifying things that by their nature are not theoretically (mathematically) measurable and practically (physically / experimentally) measurable, a theory that unfortunately, cannot be confirmed with instruments of statistics, classical logic, modal logic, system theory, measure theory and topology. On the contrary, formula (17) shows there is at least one way of consistently expressing an agreement point for all these sciences/theories 
(including fuzzy logic) while maintaining $\sigma$-additivity condition. The lack of interconnection between probability theory and fuzzy logic evidenced here when fuzzy logic accepts the weakening of the $\sigma$-additivity condition is just the small empty part of a bottle of very old wine: given the huge diversity of fuzzy membership assignments and the natural parity that exists between at least a part of them and the continuous distributions, a real explosion of new probability distribution models is expected to happen in the years to come, marking a point from where fuzzy logic and statistics will further develop side-by-side exactly because of the bridge established in between them by the $\sigma$-additivity condition.

\subsection{Negation of fuzzy membership}

As far as we know, expressing probable events (like those represented in Figure 2) as fuzzy membership (Figure 5) is a matter of interpretation, a matter of rewriting some facts from a dialect of statistics to a dialect of fuzzy logic using a rewriting rule, for example formula (17) - in our specific case considered here. Therefore, in this context, the negation operates at a semantic level: for example, if 0.5 is interpreted as a certain imposter score - and this interpretation is allowed by formula (17) because $\operatorname{FM}(0.5)=1$ (despite the fact that the statistics of experimental data says nothing more than 0.5 score is the most probable imposter score), then logically, should be impossible for the same 0.5 score to be interpreted as a genuine score, fact that is indeed true, despite some false appearances in Figure 2 and Figure 5. Indeed, the index of genuine pairs can be intoxicated accidentally by wrong segmentation results or by the impossibility that the implemented recognition system to deal successfully with the variability of acquired iris instances. This is also another facet of the imperfect experimental frameworks where the job of solving apparently conflicting information obtained through interpretation of experimental data must be done carefully by a qualified human operator / system administrator.

\subsection{Blind negation of fuzzy membership}

If no attention is given to the semantic of actually implemented system and to the actual input-output relation, negation can be made by applying fuzzy complement [29]. For example, if FM is defined by (17) as the fuzzy membership of all scores within $[0,1]$ interval with respect to the imposter distribution, 1-FM is a complementary fuzzy membership assignment defining a sort of negation in which a single element within $[0,1]$ interval is certainly a non-imposter score, namely 0.5 - the mean of the imposter distribution. This fact is, of course, counter-intuitive with respect to the practical problem. Besides, we are talking again about many values situated outside the set of experimentally observed imposter scores. This operation is meaningless with respect to the actual implemented system. This is why we called this sort of negation, blind negation. 
On the contrary, if we remember that negation by complement should have a meaning with respect to the practical problem (implemented system) and should make a real sense inside the set of reachable scores, the situation changes completely: since f-imposter and f-genuine are two fuzzy granules covering the set of reachable scores, the assertion 's is an f-imposter score' means accordingly to the actual experimental data that $\mathrm{s}$ is the Gaussian distributed number as illustrated in Figure 2 (i.e. is 0.5 with maximal probability, 0.51 with other probability and so on) whereas its negation should be 's is an f-genuine score' whose interpretation should be derived by analogy from the previous one.

\section{Negation in biometrics}

The important remark to make here is that since the fuzzy membership assignment is an interpretation of data, there is not a unique way of negating it, as already exemplified above. Even at this stage it is not capitally important to find a negation operator meaningful with respect to the practical problem, we will try this in a future paper, eventually. Bell that saved us this time is the fact that the final stage in obtaining a practicable crisp decisional model from the fuzzy model within Figure 5 is defuzzification of f-imposter and f-genuine fuzzy sets to classical disjoint intervals labeled 'imposter' and 'genuine' and partitioning $[0,1]$ interval.

\subsection{Negation as a Boolean algebraic operator}

After defuzzification, the Boolean logic of recognition can be expressed through isomorphism with the Boolean algebra generated by the empty set, $[0,1]$ interval and the two intervals 'imposter' and 'genuine', whereas negation is simply a transcript of complement operation within this Boolean algebra. This is a way of implementing a binary recognition function and a binary decisional model for biometrics, a model in which the recognition error rates are hopefully stationary (there is no proof for that, whereas increased recognition errors over time is already documented under the wrong name of "template ageing" (critical analyses of this concept can be found also in [6] and [9]). A ternary recognition function and a ternary decisional model for biometrics is obtained if the two intervals 'imposter' and 'genuine' are separated by a third one labeled 'uncertain' and covering ambiguous score values.

By investigating the consistency of the concepts of "template ageing" and "biometric menagerie" [12], [23] we found an improved quinary recognition function and a quinary decisional model for iris biometrics, which is obtained while practicing iris recognition on intelligent iris verifier systems with stored digital identities [22]. In such systems, it is possible that the lowest scores are 
imposter scores, followed by a class of degraded imposter scores obtained by dishonest users when claiming (actively hunting) different identities than they actually have (hyena), followed by a class of uncertain scores centered in 0.5 , and further to the right by a class of degraded genuine scores (goats). The rightmost class is that of genuine scores. All of these models are illustrated in Figure 6, whereas the binary and the ternary models are applicable also for classical statistical iris recognition (Figure 2, Figure 5).

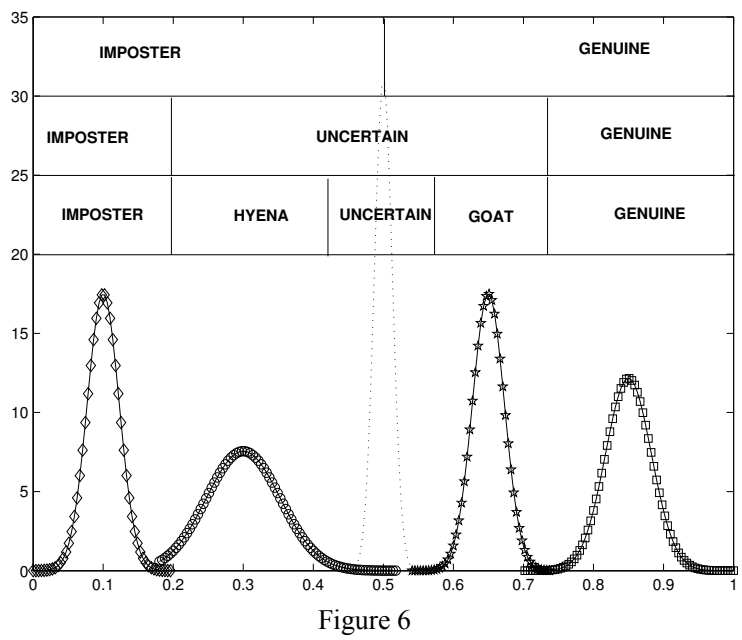

A better machine-precisiation of statistical iris recognition with distant imposter and genuine score classes; From top to bottom: binary, ternary, respectively quinary decisional models.

For all decisional models within Figure 6, negation is expressed within a Boolean logic induced by a partial power set Boolean algebra (in a similar manner as that described in [21]): the negation of the assertion ' $s$ is an impostor score' is intuitively and naturally expressed as 's is either hyena, or uncertain, or goat, or genuine score', for example. This is why we did not insist on finding the negation operator in the previous step (prior to defuzzification). Besides, between an interpretation and a Boolean logic, the latter is clearly the appropriate context of negation.

\subsection{Strong negation in biometrics}

Blind negation of fuzzy membership by fuzzy complement (see section 3.6) is a negation practiced in the codomain of the fuzzy membership function. On the contrary, the negation by complement applied to individual recognition scores s,

$\mathrm{N}(\mathrm{s})=1-\mathrm{s}$,

acts in the domain of that fuzzy membership function. The negation $\mathrm{N}$ in (18) satisfies boundary condition $\mathrm{N}(0)=1$, is continuous, involutive and decreasing with 
respect to the score s, hence it is a strong negation [7], as all instances of Sugeno $\lambda$ - complement [26], [32]. The effect of this negation is a rewriting of all recognition results from terms of similarity (proximity) in terms of non-similarity (distance). For example, applying the strong negation (18) for all available Hamming distance scores, expresses the same experimental results in terms of Hamming similarity (the complement of Hamming distance) and vice versa.

Graphically, the effect of this negation is that the score distributions are all symmetrized against the vertical pointing in 0.5 , regardless how many they are $(2$, 3 or 5). Hence, strong negation on all recognition scores has a meaningful effect with respect to the practical problem of recognition. However, the appropriate context of applying it is prior to defuzzification. After defuzzification, depending on the number of values chosen for the recognition function, the decisional model is still binary, ternary or quinary, but the order of the intervals reverses: genuine, imposter - for the binary case, genuine, uncertain, imposter - for the ternary case, and finally, genuine, goat, uncertain, hyena, imposter - for the quinary case.

\section{Conclusions}

This paper proposed a new formalization of the classical probability-possibility relation, which was further confirmed as a much complex, but natural relation between provability, observability, reachability, possibility, probability, fuzzy membership and Riemann integrability. Searching for the right context in which this relation can be consistently expressed for the particular case of experimentally obtained iris recognition results brought us inevitably to a natural (canonic) and universal fuzzification procedure - formula (17) - available for an entire class of continuously distributed random numbers, as a confluence point of statistics, classical logic, modal logic, system theory, measure theory and topology. The applications were initially intended for iris recognition scenarios and can be easily extrapolated anywhere else where there is a need of expressing the relation possibility - probability - fuzzy membership without weakening the $\sigma$-additivity condition within the definition of probability, which as this paper suggested is actually the true principle of consistent possibility-probability translation. .

\section{Acknowledgement}

This work was supported by the University of South-East Europe Lumina (Bucharest, Romania) and the Lumina Foundation (Bucharest, Romania).

\section{References}

[1] A.J. Ayer, Language, Truth, and Logic, Victor Gollancz Ltd., London, 1936.

[2] V.E. Balas, I.M. Motoc, A. Barbulescu, Combined Haar-Hilbert and LogGabor Based Iris Encoders, New Concepts and Applications in Soft Computing, Series: Studies in Computational Intelligence, vol. 417, pp. 126, Springer, 2013. 
[3] J.G. Daugman, Biometric Personal Identification System Based on Iris Analysis, U.S. Pattent 5291560 / 1991 / 1994.

[4] J.G. Daugman, How Iris Recognition Works, in Proc. 2002 IEEE Conf. on Image Processing, vol 1, pp.33-36, Rochester, New York, USA.

[5] G. Doddington, W. Liggett, A. Martin, M. Przybocki, D. Reynolds, Sheep, Goats, Lambs and Wolves: A Statistical Analysis of Speaker Performance in the NIST 1998 Speaker Recognition Evaluation, Int'l Conf. Spoken Language Processing (ICSLP), Sydney, vol. 4., pp. 1351-1354, 1998.

[6] M. Fairhurst and M. Erbilek, Analysis of physical ageing effects in iris biometrics, IET Computer Vision, 5(6):358-366, 2011.

[7] J. Fodor, Preferences and Decisions without Numbers, Online Proceedings of Budapest Tech International Jubilee Conference, 2004.

[8] P. Grother, E. Tabassi, G.W. Quinn, W. Salamon, IREX 1 Report Performance of Iris Recognition Algorithms on Standard Images, NIST, USA, 2009.

[9] P. Grother, J.R. Matey, E. Tabassi, G.W. Quinn, M. Chumakov, IREX VI: Temporal Stability of Iris Recognition Accuracy (Iris Ageing), Information Access Division, NIST, USA, July 24, 2013.

[10] L. Ma, T.N. Tan, Y.H. Wang, D.X. Zhang, Efficient iris recognition by characterizing key local variations, IEEE Transactions on Image Processing, 13(6), 739-750, 2004.

[11] S.H. Moi, H. Asmuni, R. Hassan, R.M. Othman, Multimodal biometrics: Weighted score level fusion based on non-ideal iris and face images, Expert Systems with Applications, 2014, In Press, Elsevier.

[12] I.M. Motoc, C.M. Noaica, R. Badea, C.G. Ghica, Noise Influence on the Fuzzy-Linguistic Partitioning of Iris Code Space, Soft Computing Applications, Advances in Intelligent Systems and Computing, vol. 195, pp. 71-82, Springer, 2013.

[13] N. Popescu-Bodorin, Exploring New Directions in Iris Recognition, in proc. $11^{\text {th }}$ Intl. Sym. on Symbolic and Numeric Algorithms for Scientific Computing, Conference Publishing Services - IEEE Computer Society, pp. 384-391, September 2009.

[14] N. Popescu-Bodorin, A Fuzzy View on k-Means Based Signal Quantization with Application in Iris Segmentation, $17^{\text {th }}$ Telecommunications Forum, University of Belgrade, November 2009.

[15] N. Popescu-Bodorin, L. State, Cognitive Binary Logic - The Natural Unified Formal Theory of Propositional Binary Logic, CoRR abs/1106.2352, 2010.

[16] N. Popescu-Bodorin, V.E. Balas, Comparing Haar-Hilbert and Log-Gabor based iris encoders on Bath Iris Image Database, $4^{\text {th }}$ Intl. Workshop on Soft Computing Applications, pp. 191-196, IEEE Press, July 2010.

[17] N. Popescu-Bodorin, V.E. Balas, From Cognitive Binary Logic to Cognitive Intelligent Agents, Intelligent Engineering Systems (INES), 2010 $14^{\text {th }}$ International Conference on, pp.337-340, 5-7 May 2010. 
[18] N. Popescu-Bodorin, Online CCBL Demonstrator, Applied Computer Science Testing Lab, http://lmrec.org/bodorin/ccbl/, 2011.

[19] N. Popescu-Bodorin, V.E. Balas, Exploratory Simulation of an Intelligent Iris Verifier Distributed System, Proc. $6^{\text {th }}$ IEEE Intl. Symposium on Applied Computational Intelligence and Informatics, pp. 259 - 262, IEEE Press, June 2011.

[20] N. Popescu-Bodorin, V.E. Balas, I.M. Motoc, Iris Codes Classification Using Discriminant and Witness Directions, Proc. $5^{\text {th }}$ IEEE Intl. Symp. on Computational Intelligence and Intelligent Informatics, pp. 143-148, IEEE Press, 2011.

[21] N. Popescu-Bodorin, V.E. Balas, I.M. Motoc, 8-Valent Fuzzy Logic for Iris Recognition and Biometry, Proc. $5^{\text {th }}$ IEEE Intl. Symp. on Computational Intelligence and Intelligent Informatics, (Floriana, Malta, September 1517), pp. 149-154, IEEE Press, 2011.

[22] N. Popescu-Bodorin, V.E. Balas, Learning Iris Biometric Digital Identities for Secure Authentication. A Neural-Evolutionary Perspective Pioneering Intelligent Iris Identification, Recent Advances in Intelligent Engineering Systems, pp. 409-434, vol. 378, Series: Studies in Computational Intelligence, Springer, 2012.

[23] N. Popescu-Bodorin, V.E. Balas, I.M. Motoc, The Biometric Menagerie - A Fuzzy and Inconsistent Concept, Soft Computing Applications, Advances in Intelligent Systems and Computing, vol. 195, pp. 27-43, Springer, 2013.

[24] H. Proenca, L.A. Alexandre, Toward Covert Iris Biometric Recognition: Experimental Results From the NICE Contests, IEEE Transactions on Information Forensics and Security 7(2), 798-808, 2012.

[25] P. Radu, K. Sirlantzis, G. Howells, S. Hoque, F. Deravi, A Multialgorithmic Colour Iris Recognition System, Advances in Intelligent Systems and Computing Volume 195, 2013, pp 45-56.

[26] M. Sugeno, Fuzzy measures and fuzzy integrals: A survey, in Gupta et al., Fuzzy Automata and Decision Processes, North-Holland Publisher, Amsterdam, 1977, pp. 89-102.

[27] A.M. Turing, Computing machinery and intelligence, Mind, 59, 433-460, 1950.

[28] N. Yager, T. Dunstone, The Biometric Menagerie, IEEE Transactions on Pattern Analysis and Machine Intelligence, vol. 32, no. 2, February 2010, pp. 220 - 230, ISSN 0162-8828, doi 10.1109/TPAMI.2008.291.

[29] L.A. Zadeh, Fuzzy Sets, Information and Control, 8, 338-353, 1965.

[30] L.A. Zadeh, Fuzzy sets as a basis for, a theory of possibility, Fuzzy Sets and Systems, nr. 1 /1978, pp. 3-28, North-Holland Publishing Company.

[31] L.A. Zadeh, Is there a need for fuzzy logic?, Information Sciences, nr. 178/2008, pp. 2751-2779, Elsevier, 2008.

[32] D. Dubois, H. Prade, Fuzzy sets and systems: theory and applications, volume 144, Mathematics in Science and Engineering Series, Academic Press Inc., 1980, London, UK. 